\title{
Agronomic and genetic analysis of Suweon 542, a rice floury mutant line suitable for dry milling
}

\author{
Young-Jun $\mathrm{Mo}^{1 \dagger}$, Ji-Ung Jeung ${ }^{1{ }^{*}}$, Young-Seop Shin ${ }^{1}$, Chul Soo Park², Kyung-Ho Kang ${ }^{1}$ and Bo-Kyeong Kim ${ }^{1}$
}

\begin{abstract}
Background: Producing rice flour of good quality by dry milling is necessary to reduce milling costs and promote the processed rice food industry. This study was conducted to evaluate the dry milling properties of Suweon 542, a floury endosperm mutant, and identify the chromosomal region responsible for the floury endosperm characteristics.

Results: Compared with the wild type, after dry milling process, the grain hardness of Suweon 542 was significantly lower because of its round and loosely packed starch granules. Also, the flour of Suweon 542 had significantly smaller particles and less damaged starch than Namil and other rice cultivars and its particle size distribution was similar to a commercial wheat cultivar. Considering that the yield loss of Suweon 542 due to its floury endosperm was largely compensated for by an increased number of spikelets per panicle, Suweon 542 has potential value as a raw material for rice flour production. Association analysis using 70 genome-wide SSR markers and $94 \mathrm{~F}_{2}$ plants derived from Suweon 542/Milyang 23 showed that markers on chromosome 5 explained a large portion of the variation in floury grains percentage (FGP). Further analysis with an increased number of SSR markers revealed that the floury endosperm of Suweon 542 was directed by a major recessive locus, flo7 $(t)$, located in the 19.33-19.86 Mbp region of chromosome 5, with RM18639 explaining 92.2\% of FGP variation in the $F_{2}$ population.

Conclusions: The floury endosperm of Suweon 542 is suitable for dry milling, with a small flour particle size and low damaged starch content. Further physical mapping of flo7(t), the floury endosperm locus of Suweon 542, would facilitate efficient breeding of rice cultivars with proper dry milling adaptability that can be used in the processed rice food industry.
\end{abstract}

Keywords: Rice; Mutation; Sodium azide; Starch; Floury endosperm; Dry milling

\section{Background}

Rice is one of the largest crops grown in the world and is a staple food for over approximately one-half of the world population especially in Asian countries (Singh et al. 2005). Rice is usually consumed as cooked rice and relatively a small amount is used to make ingredients for process (Zhou et al. 2002), while recently rice flour is becoming attractive as raw material of a substitute for wheat flour in terms of 'gluten-free' (Demirkesen et al. 2013), or potato-based French-fry (Kadan et al. 2001). In Korea, only $6 \%$ of the total produced rice is used as raw materials for processed food production, although there

\footnotetext{
* Correspondence: jrnj@korea.kr

${ }^{\dagger}$ Equal contributors

'National Institute of Crop Science, Rural Development Administration, Suwon 441-857, Republic of Korea

Full list of author information is available at the end of the article
}

are more than 300 types of processed food made from rice (Kim 2011).

Rice consumption has been continuously decreasing as the eating habits of Koreans have become westernized and diversified. The per capita annual rice consumption in Korea has dropped sharply from $136.4 \mathrm{~kg}$ in 1970 to $69.8 \mathrm{~kg}$ in 2012 (Statistics Korea 2013). The Korean government, therefore, has been trying to promote rice consumption by invigorating the processed food industry using rice flour. The status of rice consumption in Japan is also very similar to Korea, for which application of rice flour for bread making has been considered as one approach to increase rice consumption (Araki et al. 2009).

To facilitate the market for processed rice foods, it is essential to develop proper milling technology in terms of flour particle size and damaged starch content to produce high quality rice flour at competitive cost 
(Hasjim et al. 2013). Dry milling and wet milling are the two major processes used to produce rice flour. Although the dry milling process is relatively simple with a lower production cost, damaged starch content increases because of the high grain hardness of rice. In wet milling, the quality of rice flour is improved by reducing flour particle size as well as damaged starch content through soaking procedures. However, the production costs are high because of the additional expenses associated with the disposal of waste water, sterilization and drying of the wet flour (Altheide et al. 2012; Jun et al. 2008; Kim et al. 2009; Lee and Kim 2011). Recently developed technologies such as jet milling and cryogenic milling also require expensive investment and production (Ashida et al. 2010; Tran et al. 2011). Therefore, developing new rice cultivars with dry milling adaptability as well as good processing properties is an important goal of rice breeding in Korea.

Mutagenesis approaches using chemicals or irradiation have been widely used to diversify grain quality in rice. Satoh and Omura (1981) induced various endosperm mutants such as 'waxy', 'dull', 'floury', and 'sugary' using ethyl methanesulfonate (EMS), ethylene imine (EI), and Nmethyl-N-nitrosourea (MNU). In Korea, MNU treatment of the rice variety Ilpum was successfully used by the Rural Development Administration to develop new endosperm mutant cultivars such as Seolgaeng with opaque endosperm, Baegjinju with dull endosperm, Goami 2 with resistant starch, and Keunnun with giant embryos (Hong et al. 2011; Hong et al. 2012a, 2012b). The Korea Atomic Energy Research Institute has released 10 rice cultivars including Nogwonchal, a glutinous cultivar, using radiation such as gamma rays (Kang et al. 2008). Also, Shin et al. (2009) used sodium azide (SA) to develop endosperm mutant lines with waxy, dull, white-core, or floury characteristics. Among endosperm mutants such as floury, white-core or opaque kernel phenotypes, floury mutants have a white opaque grain appearance similar to waxy and dull mutants but are stained blue-black by I-KI solution and have loosely packed starch granules that break easily (Satoh and Omura 1981). The endosperm of rice floury mutants, therefore, has more suitable physical characteristics for milling processes than normal non-glutinous lines or waxy and dull mutants in terms of the grain hardness and amylose content, respectively.

Genetic loci associated with floury endosperm in rice were previously reported on chromosomes $1,3,4,5$, and 8. The flo- 1 and flo-2 mutants were induced by MNU treatment of the rice cultivar Kinmaze and their loci were localized to chromosomes 5 and 4, respectively (Satoh and Omura 1981; Kaushik and Khush 1991). She et al. (2010) identified the gene responsible for the floury endosperm of the flo-2 mutant, FLOURY ENDOSPERM2 (FLO2), which mediates protein-protein interactions. flo-3 is a floury endosperm mutant with a lower level of the 16$\mathrm{kDa}$ allergenic protein and its locus is on chromosome 3 (Nishio and Iida 1993). flo4 and flo5 are T-DNA insertion mutant lines in OsPPDKB on chromosome 5 and OsSSIIIa on chromosome 8 , respectively, that also show a floury endosperm with loosely packed starch granules (Kang et al. 2005; Ryoo et al. 2007). flo(a) was induced by MNU treatment of the cultivar Hwacheong and was identified as OsTPR on chromosome 4 (Qiao et al. 2010). flo6 was induced by EMS treatment of the cultivar Nipponbare and the responsible sequence variation was identified as a G/A SNP in OsAGPL2-3 on chromosome 1 (Zhang et al. 2012). Most previous studies on rice floury endosperm loci have focused on the function of identified genes while few studies have examined the practical usage of floury endosperm in terms of flour production and agricultural productivity.

In this study, we evaluated the dry milling suitability of Suweon 542 as well as its major agronomic traits related with yield potential and crop rotation systems in Korea. Genetic analysis was also carried out to identify the chromosomal location responsible for the floury endosperm characteristics of Suweon 542 to establish a basis for molecular breeding to develop rice cultivars with high quality flour.

\section{Results}

Agronomic traits and grain characteristics of Suweon 542 The major agronomic and yield-related traits of Suweon 542 were evaluated along with its wild type cultivar, Namil (Table 1). The heading date of Suweon 542 was 3 days later than Namil. While the culm length of Suweon 542 was $6 \mathrm{~cm}$ taller compared with the wild type, there was no significant difference in panicle length. Brown rice (dehulled kernel) yield per ha was 0.330 tons lower in Suweon 542 than in Namil, mainly because of the low ripened grains percentage and grain weight of the floury endosperm seeds. However, the number of spikelets per panicle was significantly increased in Suweon 542, which is likely to have compensated for a considerable amount of the yield loss caused by the floury endosperm characteristics.

Table 1 Major agronomic traits of Suweon 542 in comparison with its wild type, Namil

\begin{tabular}{|c|c|c|c|c|c|c|c|c|}
\hline Line & $\begin{array}{c}\text { HD } \\
\text { (days) }\end{array}$ & $\begin{array}{c}\mathrm{CL} \\
(\mathrm{cm})\end{array}$ & $\begin{array}{c}\text { PL } \\
(\mathrm{cm})\end{array}$ & $\begin{array}{c}\text { TN } \\
\text { (No.) }\end{array}$ & $\begin{array}{c}\text { SN } \\
\text { (No.) }\end{array}$ & $\begin{array}{l}\text { RGP } \\
(\%)\end{array}$ & $\begin{array}{c}\text { TGW } \\
(g)\end{array}$ & $\begin{array}{c}\text { BRY }^{\mathrm{a}} \\
\text { (ton/ha) }\end{array}$ \\
\hline Namil & $101^{b}$ & $78^{b}$ & $25^{a}$ & $11^{a}$ & $117^{b}$ & $87^{a}$ & $26.5^{a}$ & $6.00^{a}$ \\
\hline Suweon 542 & $104^{a}$ & $84^{a}$ & $26^{a}$ & $11^{a}$ & $155^{\mathrm{a}}$ & $58^{b}$ & $22.2^{\mathrm{b}}$ & $5.67^{b}$ \\
\hline
\end{tabular}

${ }^{a}$ Means with the same letter are not significantly different at $P<0.05$ in the least significant difference test $\left(\mathrm{LSD}_{0.05}\right)$. The means of each line were obtained from replicated yield trials with three replication plots. HD: days-to-heading after sowing, CL: culm length, PL: panicle length, TN: tiller number, $\mathrm{SN}$ : spikelet number per panicle, RGP: ripened grains percentage, TGW: 1,000-grain weight of brown rice (dehulled kernel), BRY: brown rice yield. 
The grain and endosperm appearance of Suweon 542 was also examined in comparison with its wild type cultivar (Figure 1). While Namil showed transparent endosperm as a typical non-glutinous rice, the endosperm of Suweon 542 had chalky texture with white opaque appearance (Figure 1A). Cross-sectional observation of dehulled kernels revealed that most part of the Suweon 542 endosperm was white opaque except for a thin peripheral area of the grain (Figure 1B). Scanning electron microscopy showed that Suweon 542 has loosely packed starch granules with irregular and rounded shape while Namil has more densely packed starch granules with polyhedral angles (Figure 1C).

\section{Dry milling properties of Suweon $\mathbf{5 4 2}$}

The suitability of Suweon 542 as a raw material for high quality dry milled rice flour was evaluated. The grain hardness of Suweon 542's dehulled kernel was only about $45 \%$ of the wild type cultivar Namil (Table 2). The dehulled kernel of Suweon 542 was significantly softer than even Seolgaeng, a Korean rice cultivar with opaque endosperm, demonstrating that Suweon 542 could be easily pulverized during the milling process.

Particle size and distribution in the dry milled rice flour were measured by laser diffraction analysis. The mean particle size of Suweon 542 was $82.0 \mu \mathrm{m}$, which was significantly smaller than Selogaeng $(97.6 \mu \mathrm{m})$, Namil $(109.1 \mu \mathrm{m})$ and Hwaseong $(112.2 \mu \mathrm{m})$ (Table 2). The particle size distribution displayed in Table 3 shows that Suweon 542 had a much greater fraction of fine particles compared with the other rice cultivars. The flour particle size distribution of Suweon 542 was similar to that of Keumkang, a commercial Korean wheat cultivar, demonstrating its suitability for producing fine flour by dry milling. The damaged starch content of the dry milled flour was $4.9 \%$ in Suweon 542, which was significantly lower than in Seolgaeng (7.1\%),

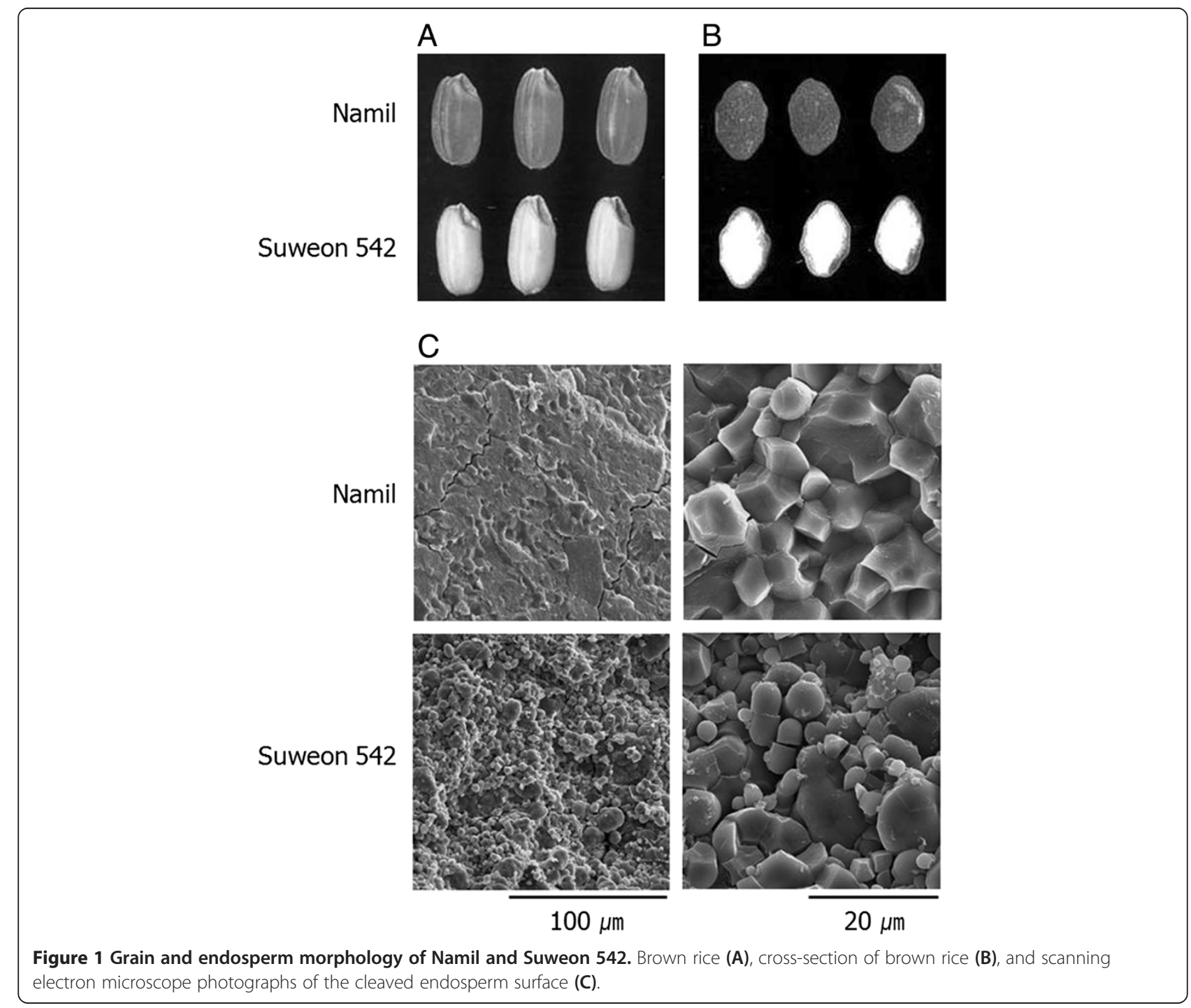


Table 2 Physicochemical properties of grains and rice flours

\begin{tabular}{|c|c|c|c|c|c|c|c|}
\hline \multirow[t]{2}{*}{ Line } & \multicolumn{7}{|c|}{ Rice flour properties } \\
\hline & $\begin{array}{c}\text { Grain hardness } \\
(\mathrm{Kg})\end{array}$ & $\begin{array}{c}\text { Mean particle size } \\
(\mu \mathrm{m})\end{array}$ & $\begin{array}{c}\text { Damaged starch } \\
(\%)\end{array}$ & $\begin{array}{l}\text { Lightness } \\
\text { (CIE value) }\end{array}$ & Ash (\%) & Protein (\%) & Amylose (\%) \\
\hline Hwaseong & $7.8 \pm 1.60$ & $112.2 \pm 0.4$ & $10.3 \pm 0.2$ & $88.6 \pm 0.01$ & $0.84 \pm 0.02$ & $7.5 \pm 0.16$ & $18.5 \pm 0.2$ \\
\hline Seolgaeng & $5.9 \pm 0.87$ & $97.6 \pm 1.6$ & $7.1 \pm 0.1$ & $90.3 \pm 0.06$ & $0.72 \pm 0.01$ & $6.6 \pm 0.11$ & $17.5 \pm 0.6$ \\
\hline Namil & $7.5 \pm 2.21$ & $109.1 \pm 0.6$ & $9.2 \pm 0.2$ & $88.7 \pm 0.09$ & $0.82 \pm 0.01$ & $9.2 \pm 0.25$ & $17.7 \pm 0.3$ \\
\hline Suweon 542 & $3.3 \pm 0.48$ & $82.0 \pm 0.6$ & $4.9 \pm 0.1$ & $90.0 \pm 0.07$ & $0.79 \pm 0.01$ & $7.5 \pm 0.15$ & $18.5 \pm 0.3$ \\
\hline
\end{tabular}

Namil (9.2\%), and Hwaseong (10.3\%) (Table 2). It should be noted that damaged starch content was measured with finer flour particles in Suweon 542 compared with the other cultivars. If the three rice cultivars had been milled to a finer particle size, similar to that of Suweon 542, it is plausible that they would have had even higher damaged starch content.

The flour physicochemical properties such as lightness and ash, protein and amylose contents were also measured to identify the distinct characteristics of Suweon 542 (Table 2). The flour lightness of Suweon 542 was significantly higher than Namil and Hwaseong and similar to Seolgaeng. On the other hand, the ash content of Suweon 542 was significantly lower than Namil and Hwaseong, while significantly higher than Seolgaeng. Compared with the wild type, protein content was significantly lower while amylose content was significantly higher in Suweon 542. Despite these significant differences, the overall physicochemical properties of the Suweon 542 flour were within the range of normal nonglutinous rice cultivars, except for its markedly low grain hardness due to the floury endosperm.

Inheritance of floury endosperm in the $F_{2}$ population To understand the mode of inheritance of the floury endosperm characteristics of Suweon 542, a population composed of $94 \mathrm{~F}_{2}$ plants was developed from a cross between Suweon 542 and Milyang 23, a Tongil-type cultivar.

As the phenotypic data for genetic analysis, floury grains percentage (FGP) was evaluated by using randomly selected 150-200 dehulled kernels (Figure 2A). All kernels of Suweon 542 exhibited typical floury characteristics $(\mathrm{FGP}=100 \%)$. Grain endosperm chalkiness of rice is a varietal characteristic as some kernels of Milyang 23 had significant white opaque area (Figure 2A). Although the appearance of chalky kernel in Milyang 23 was distinguishable from floury endosperm of Suweon 542 (Figure 2A), we classified the kernels with more than $50 \%$ white opaque area as 'floury' during the visual inspection on the $\mathrm{F}_{2: 3}$ seeds. Based on the criterion, the FGP of Milyang 23 was $8.8 \%$.

We have evaluated $94 \mathrm{~F}_{2}$ individuals in terms of FGP by using the $\mathrm{F}_{2: 3}$ seeds (Figure $2 \mathrm{~B}$ ). The frequency distribution of FGP exhibited an abnormal distribution pattern (skewness $=1.05, \quad w=0.97 ; \quad P<0.0001$ ), with 23 individuals $(24.5 \%)$ showed FGP $<10 \%$ while 16 individuals $(17.0 \%)$ showed FGP $>90 \%$. Most of the remaining 55 F2 individuals (58.5\%) had FGP of 15\%-35\%. Therefore, it was inferred that the floury endosperm of Suweon 542 is controlled by a major recessive genetic factor. The monogenic recessive nature of floury endosperm was also strongly supported as the 55 heterogeneous $F_{2}$ individuals segregated in a 3 normal: 1 floury ratio, where the maximum $\chi^{2}$ value was $4.81(0.05<\mathrm{P}<0.01)$. Instead of inferring genotypes of segregating progenies at the floury locus by using the estimated FGP, we conducted association analysis between the FGP and marker genotypes of $F_{2}$ individuals by using single-locus ANOVA.

\section{Mapping of the floury endosperm locus in Suweon 542}

A linkage map skeleton was constructed using 70 SSR markers that were polymorphic between Suweon 542 and Milyang 23 and evenly distributed across the 12 rice chromosomes, with 4-8 markers per chromosome (data

Table 3 Comparison of particle size distribution patterns of rice flours produced by the dry milling method

\begin{tabular}{|c|c|c|c|c|c|c|}
\hline \multirow[t]{2}{*}{ Line $^{a}$} & \multicolumn{6}{|c|}{ Mean particle size $(\mu \mathrm{m})$ on the relative proportions of size fraction ${ }^{b}$} \\
\hline & Over-all & $<10 \%$ & $<25 \%$ & $<50 \%$ & $<75 \%$ & $<90 \%$ \\
\hline Hwaseong & $112.2 \pm 0.4$ & $45.4 \pm 0.8$ & $78.9 \pm 0.6$ & $114.1 \pm 0.7$ & $146.1 \pm 0.2$ & $174.3 \pm 0.3$ \\
\hline Seolgaeng & $97.6 \pm 1.6$ & $30.7 \pm 2.1$ & $62.1 \pm 2.0$ & $98.4 \pm 1.5$ & $131.5 \pm 1.3$ & $160.0 \pm 1.3$ \\
\hline Namil & $109.1 \pm 0.6$ & $44.1 \pm 0.5$ & $77.6 \pm 0.7$ & $111.6 \pm 0.7$ & $141.9 \pm 0.4$ & $168.3 \pm 1.1$ \\
\hline Suweon 542 & $82.0 \pm 0.6$ & $17.4 \pm 0.5$ & $38.7 \pm 0.6$ & $82.2 \pm 0.6$ & $118.6 \pm 0.6$ & $147.6 \pm 1.2$ \\
\hline Keumkang & $91.0 \pm 0.5$ & $21.8 \pm 0.3$ & $48.2 \pm 0.6$ & $92.4 \pm 0.4$ & $129.6 \pm 0.6$ & $159.4 \pm 1.1$ \\
\hline
\end{tabular}

${ }^{\mathrm{a} A}$ Korean wheat cultivar, Keumkang, was included as a control.

${ }^{\mathrm{b}}$ Standard deviations are indicated followed by mean particle size. 


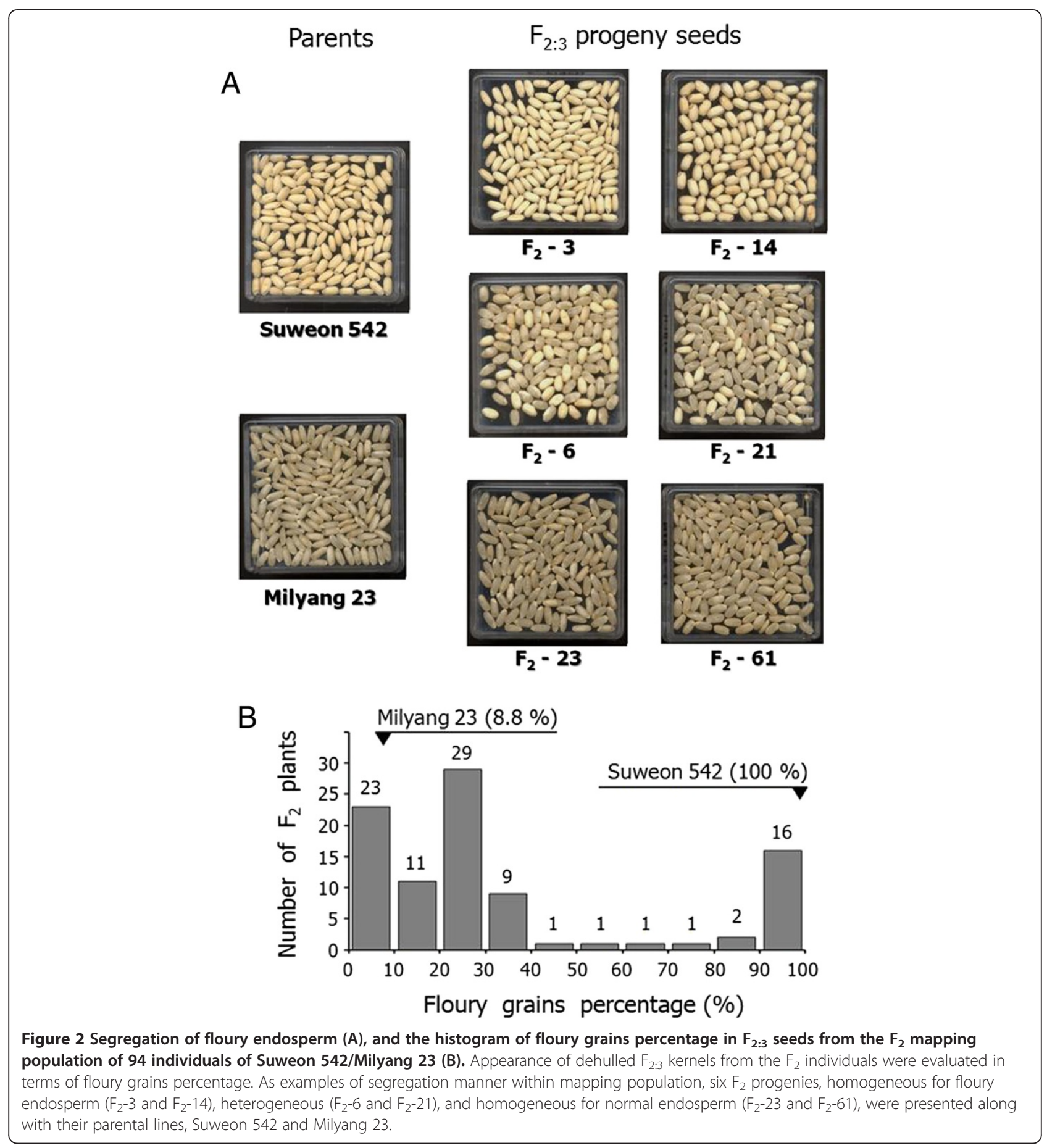

not shown). Association analysis, by using single-locus ANOVA, was conducted to estimate the allelic effect of each SSR locus on the variation in FGP of the $94 F_{2}$ plants derived from Suweon 542/Milyang 23.

Among 6 SSR markers on the linkage map skeleton of chromosome 5 (Table 4; Locus, Ap $=1$ ), a significant $F$ value with high $R^{2}$ was detected at two SSR loci; RM5844 at the 9.15 Mbp region $\left(R^{2}=0.463\right)$ and $\mathrm{RM} 3351$ at the
$20.76 \mathrm{Mbp}$ region $\left(R^{2}=0.520\right)$, where the allele type of Suweon 542 was favorable to increase FGP (Table 4; Genetic effect). Although significant SSR loci were also detected on chromosomes 7 (RM1243, $R^{2}=0.079$ ), 11 (RM5961, $\left.R^{2}=0.064\right)$, and 12 (RM8215, $\left.R^{2}=0.150\right)$, they were less effective than the genetic factor(s) on chromosome 5. Out of 70 SSR loci on the linkage map skeleton, related with the FGP of Milyang 23 (8.8\%), RM8215 was 
Table 4 Summary of association analyses between FGP of $F_{2: 3}$ seeds and SSR genotypes on chromosome 5

\begin{tabular}{|c|c|c|c|c|c|c|c|c|c|c|c|c|c|c|c|c|c|}
\hline \multicolumn{2}{|l|}{ Locus $^{a}$} & \multicolumn{4}{|c|}{ Mirror-map information ${ }^{\mathbf{b}}$} & \multicolumn{4}{|c|}{ Segregation test ${ }^{c}$} & \multicolumn{3}{|c|}{ Genotype mean ${ }^{d}$} & \multicolumn{2}{|c|}{ ANOVA $^{\mathrm{e}}$} & \multicolumn{3}{|c|}{ Genetic effect $^{f}$} \\
\hline Marker & Ap & Size (bp) & Mbp & BAC/PAC & cM & A & $\mathrm{H}$ & B & $x^{2}$ & A & $\mathrm{H}$ & B & $F$ & $R^{2}$ & Add & Dom & DeD \\
\hline RM5693 & 1 & 200 & 0.46 & OSJNBa0068N01 & 4.6 & 18 & 59 & 17 & 5.4 & 44.4 & 34.2 & 23.6 & 1.6 & & & & \\
\hline M3322 & 1 & 121 & 4.26 & P0685E10 & 32.0 & & 44 & 17 & 3.8 & 52.0 & 30.5 & & 8.7 & 0.164 & & & 0.1 \\
\hline M3193 & 2 & 126 & 4.97 & $\mathrm{P} 0453 \mathrm{H} 11$ & 36.4 & & 48 & 17 & 2.6 & 56.3 & 29.3 & 10.4 & 13.6 & 0.231 & -23.0 & -4.1 & 0.1 \\
\hline M5844 & 1 & 195 & & OSJNBa0032D15 & $53.5 \sim 54.3$ & 30 & 40 & 24 & 2.4 & 66.3 & 25.7 & 8.1 & 39.3 & 0.463 & -29.1 & -11.5 & 0.3 \\
\hline M3838 & 2 & 198 & 16.55 & OSJNBa0077J17 & 60.7 & 28 & 39 & 26 & 2.0 & 63.0 & 26.3 & 15.4 & & & & & 0.5 \\
\hline M6024 & 2 & 178 & 17.81 & OJ1118_F06 & 67.5 & 25 & 41 & 27 & 1.1 & 75.6 & 26.0 & 7.1 & 70.8 & 0.612 & -34.3 & & 0.4 \\
\hline M18614 & 2 & 164 & 19.22 & OSJNBa0014C03 & $73.9 \sim 75.0$ & 22 & 51 & 21 & 0.5 & 81.9 & 27.0 & 1.8 & 101.7 & 0.691 & -40.1 & -14.9 & 0.3 \\
\hline RM164 & 2 & 265 & 19.26 & OSJNBa0014C03 & $73.9 \sim 75.0$ & 20 & 54 & 20 & 1.7 & 91.6 & 25.5 & 0.4 & 272.0 & 0.857 & -45.6 & -20.5 & 0.4 \\
\hline M18620 & 3 & 245 & 19.28 & OSJNBb0092G21 & $73.9 \sim 75.0$ & 20 & 54 & 20 & 1.7 & 91.6 & 25.5 & 0.4 & 272.0 & 0.857 & -45.6 & -20.5 & 0.4 \\
\hline RM18624 & 3 & & & OSJNBb0092G21 & $73.9 \sim 75.0$ & 20 & 54 & 20 & 1.7 & 91.6 & & & & 0.857 & -45.6 & & 0.4 \\
\hline M18639 & 3 & 317 & 19.73 & OJ1174_H11 & $75.0 \sim 77.4$ & 19 & 53 & 22 & 1.4 & 95.5 & 26.3 & 0.4 & 538.7 & 0.922 & -47.6 & -21.6 & 0.4 \\
\hline RM18648 & 3 & 296 & 19.86 & P0040B10 & $75.0 \sim 77.4$ & 18 & 53 & 23 & 1.7 & 95.2 & 27.6 & 1.7 & & 0.865 & -46.8 & & 0.45 \\
\hline RM1386 & 3 & 197 & 20.06 & P0668F02 & $75.0 \sim 77.4$ & 18 & 53 & 23 & 1.7 & 95.2 & 27.6 & 1.7 & 290.8 & 0.865 & -46.8 & -20.9 & 0.4 \\
\hline RM3351 & 1 & 134 & 20.76 & OJ1212_B02 & 80.7 & 20 & 44 & 30 & 2.1 & 77.8 & 31.1 & 9.8 & 49.2 & 0.520 & -34.0 & -12.7 & 0.3 \\
\hline RM5558 & 2 & 173 & 21.25 & OJ1301_G07 & 86.0 & 23 & 40 & 31 & 3.0 & 71.3 & 30.6 & 11.2 & 37.0 & 0.448 & -30.1 & -10.7 & 0.3 \\
\hline RM5642 & 2 & 129 & 22.25 & OJ1126_D01 & 92.0 & 22 & 38 & 33 & 5.1 & 62.4 & 31.9 & 18.4 & 14.2 & 0.240 & -22.0 & -8.5 & 0.3 \\
\hline RM3870 & 2 & 193 & 22.96 & OJ1387_F08 & 95.3 & 23 & 38 & 33 & 5.0 & 65.5 & 29.7 & 17.6 & 19.5 & 0.300 & -24.0 & -11.9 & 0.50 \\
\hline RM3476 & 1 & 132 & 23.91 & OJ1004_E02 & 101.0 & 18 & 46 & 30 & 2.6 & 62.8 & 35.6 & 15.0 & 14.3 & 0.239 & -23.9 & -3.3 & 0.1 \\
\hline RM7653 & 2 & 121 & 27.42 & OSJNBa0079H23 & 111.6 & 18 & 43 & 33 & 4.8 & 52.1 & 31.7 & 27.7 & 3.4 & 0.069 & -12.2 & -8.1 & 0.67 \\
\hline RM1054 & 1 & 150 & 29.23 & OJ1007_H05 & 122.0 & 23 & 41 & 30 & 2.2 & 49.8 & 28.1 & 30.6 & 3.4 & 0.069 & -9.6 & -12.1 & 1.26 \\
\hline
\end{tabular}

${ }^{a}$ DNA markers were tested in $94 \mathrm{~F}_{2}$ progenies derived from Suweon 542/Milyang 23. After conducting a first round of association analyses over well-defined 6 anchor markers (application: $A p=1)$, an additional 9 SSR markers $(A p=2)$ were applied to narrow down the putative location responsible for the floury endosperm characteristics on chromosome 5. Five SSR markers $(\mathrm{Ap}=3)$ were further applied to localize the flo7 $(t)$ nearby a BAC lone OJ1174_H11 (BenBank Acc. = AC104708.2), tagged by a SSR marker, RM18639.

${ }^{\text {b} T h e ~ e x p e c t e d ~ P C R ~ p r o d u c t ~ s i z e ~ i n f e r r e d ~ b y ~ e-L a n d i n g s ~ o n ~ a ~ r e f e r e n c e ~ r i c e ~ g e n o m e, ~ ' O s-N i p p o n b a r e-R e f e r e n c e-I R G S P-1.0 ' ~(h t t p: / / r a p d b . d n a . a f f r c . g o . j p / d o w n l o a d / \% ~}$ irgsp1.html). The cM position was directly adopted from the IRGSP Build5 Pseudomolecules (http://rgp.dna.affrc.go.jp/E/\%IRGSP/Build5/build5.html) according to the locations of BAC/PAC clones harboring priming sites for each SSR marker.

${ }^{C} \mathrm{~A}$ and $\mathrm{B}$ are homozygous for Suweon 542 and Milyang 23 allele types, respectively, and $\mathrm{H}$ indicates heterozygous progenies at the tested locus. All $X^{2}$ values for the segregation distortion test were not significant at $P<0.05$.

${ }^{\mathrm{d}}$ Mean floury grains percentage (FGP) for each genotype category revealed by the SSR markers.

e Single-locus ANOVA to test associations between SSR marker genotypes and FGP. For the $F$-test, markers having less than 0.05 for significance were declared as significant empirically $\left({ }^{*} P<0.05\right.$ and $\left.{ }^{* * *} P<0.001\right)$. The explainable variation portion of FGP at the tested locus $\left(R^{2}\right)$ is also shown.

${ }^{f}$ Additive effect (Add), dominance effect (Dom), and degree of dominance (DeD) were estimated for the significant loci: Add $=(B$ mean $-A$ mean) $/ 2$, Dom $=H$ mean - (B mean $+A$ mean) $/ 2$, and DeD $=$ Dom / Add, where $A$ and $B$ are homozygous $F_{2}$ individuals for Suweon 542 and Milyang 23 while $H$ is heterozygous individuals at the tested locus.

the only locus responsible for FGP incensement by the allele type of Milyang 23 (additive effect = 4.1).

Nine polymorphic SSR markers were applied to narrow down the putative location responsible for the floury endosperm characteristics by increasing the marker density of chromosome 5 (Table 4; Locus, Ap =2). RM164 at the 19.26 Mbp region showed the highest $F$-value explaining $85.7 \%$ of the variation in FGP of the $\mathrm{F}_{2}$ population. $F$-value decreased as the SSR markers became more distant from RM164, showing 101.7 for RM18614, which was around $40 \mathrm{Kbp}$ upstream of RM164, and 49.2 for RM3315, which was about 1.5 Mbp downstream of RM164 (Table 4; ANOVA). Therefore, we speculated that the floury endosperm locus of Suweon 542 was located at the region spanning RM18614-RM164-RM3351 on chromosome 5, which is at 19.22-20.76 Mbp of the reference rice genome, and 97.8-112.2 cM of linkage map constructed with the genotypes of $94 \mathrm{~F}_{2}$ individuals (Table 4, Figure 3A). This new recessive floury gene in Suweon 542 was tentatively designated as floury-7 (flo7).

To construct a high density linkage map for $f l o 7(t), 26$ SSR markers only for the interval between RM164 and RM3351 were selected (data not shown). Five SSR markers (RM18620, RM18624, RM18639, RM18648, and RM1386) were positive for polymorphism between Suweon 542 and Milyang 23 and segregated for marker alleles in $\mathrm{F}_{2}$ progenies (Table 4; Locus, Ap=3). However, due to the small size of mapping population $(N=94)$, RM18620 and RM18624 were mapped at the same position with RM164 (97.8 cM), and RM18648 and RM1386 also co- 


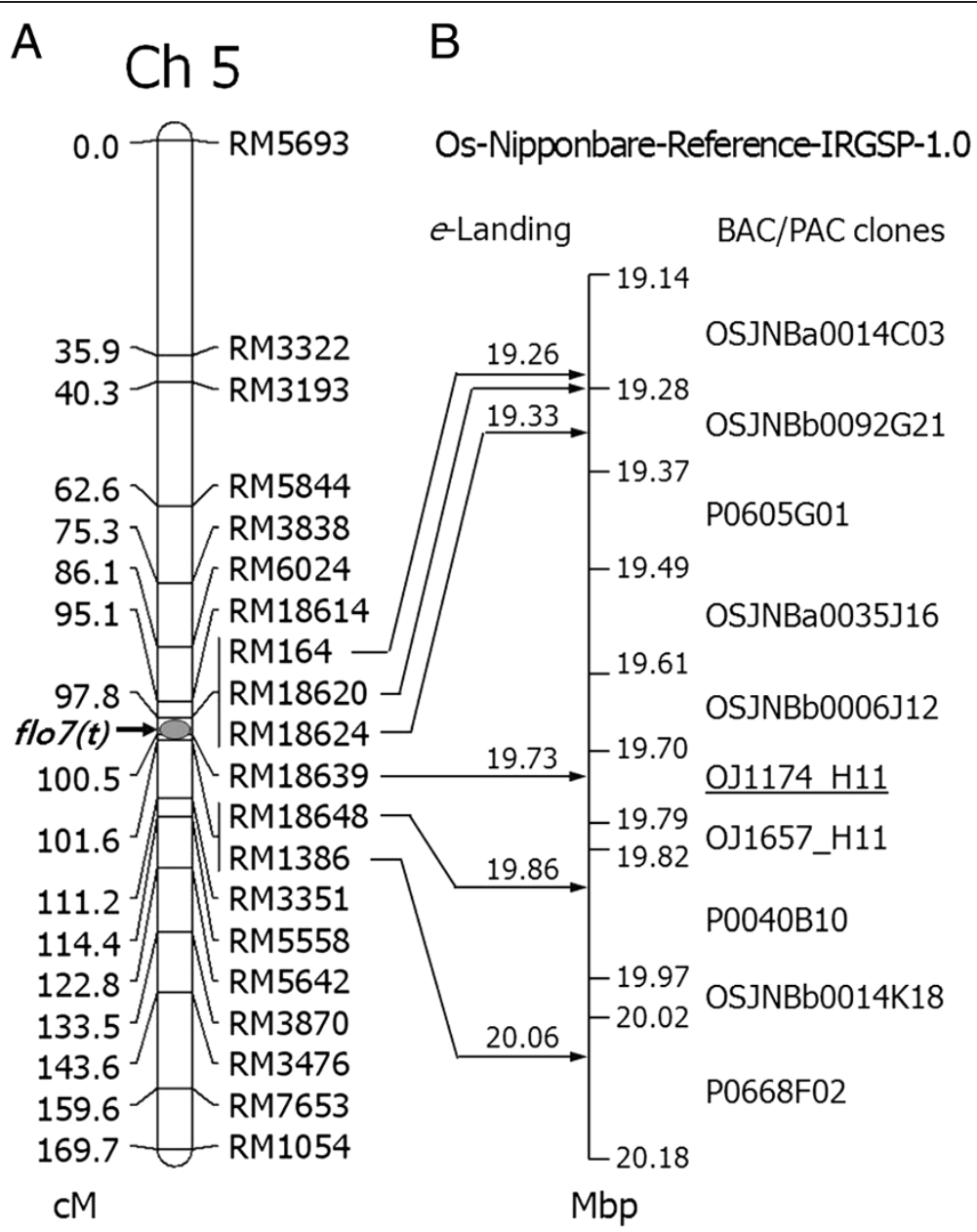

Figure 3 Localization of $f l o 7(t)$ on the linkage map of rice chromosome $5(A)$, and e-Landings of significant SSR markers on a reference rice genome to delimit the corresponding $1.04 \mathrm{Mbp}$ virtual contig (B). SSR markers were applied based on F-statistics from single-locus ANOVA results to narrow down the putative location responsible for the floury endosperm characteristics (see Table 4; ANOVA). The accumulative genetic distances shown in centiMorgans (CM) were calculated using the observed recombination fractions between SSR marker pairs in the $F_{2}$ mapping population based on the Kosambi mapping function (see also Table 4; Mirror-map information). Note that the SSR markers, placed at 97.8 CM (RM164, RM18620, RM18624) and 101.6 CM (RM18648, RM1386) were co-segregated each other in the $F_{2}$ mapping population from Suweon 542/Milyang $23(N=94)$. The $1.04 \mathrm{Mbp}$ virtual contig, composed of overlapping $10 \mathrm{BAC} / \mathrm{PAC}$ clones, was delimited by e-Landings of six significant SSR markers on the reference rice genome, 'Os-Nipponbare-Reference-IRGSP-1.0'. The underlined clone (OJ1174_H11) includes the genomic sequences corresponding to RM18639, the most significant SSR marker for the variation in FGP, and the OsPPDKB gene (Kang et al. 2005).

segregated at $101.6 \mathrm{cM}$, while RM18369 was placed at 100.5 cM (Table 4; Segregation test, Figure 3A). When association analysis was conducted again, RM18639 exhibited the highest $F$ value across the chromosome 5 and explained $92.2 \%$ of the variation in FGP of the $\mathrm{F}_{2}$ population (Table 4; ANOVA). The result also reconfirmed that the monogenic nature of a recessive floury endosperm gene, $f l o 7(t)$, which was likely to be an additive gene (degree of dominance $=0.45$ ). The estimated additive effect of RM18639 on FGP was $-47.6 \%$ (Table 4; Genetic effect), illustrating that when the genotype of Milyang 23 (FLO7/FLO7) is substituted with that of Suweon 542 (flo7/flo7), the expected FGP of Milyang 23 would be $95.2 \%$ (breeding value). Insignificant $\chi^{2}$ value from the segregation test on marker genotypes of RM18639 (1.4, $P>0.05)$ also indicated that transferring the flo 7 allele into Tongil-type or indica rice cultivars, through conventional breeding approaches, would not be hampered by serious segregation distortion (Table 4; segregation test).

$e$-Landing of each SSR marker on the reference rice genome could confirm relative orders of the co-segregating SSR markers on a $1.04 \mathrm{Mbp}$ virtual contig composed of overlapping $10 \mathrm{BAC} / \mathrm{PAC}$ clones (Figure $3 \mathrm{~B}$ ). We concluded that the intervening physical region between 19.33$19.86 \mathrm{Mbp}$ of the reference rice genome harbors the major genetic factor directing the floury endosperm characteristics of Suweon 542. Compared to the adjacent markers, 
RM18624 $(F=272.0)$ and RM18648 $(F=290.8)$, the highest $F$ value at RM18639 (538.7) strongly suggests that the most highly likely position for $f l o 7(t)$ is near $19.73 \mathrm{Mbp}$ region of the reference rice genome on a BAC clone OJ1174_H11 $($ GenBank Acc. $=$ AC104708.2) (Figure 3B).

The ANOVA located $f l o 7(t)$ in the similar chromosomal region, where flo4 was previously reported (Kang et al. 2005). They described that the mutant phenotype was generated by T-DNA or Tos 17 insertions into the $O S P P D K B$ gene. Based on the reference rice genome database, the corresponding location was determined at 19.72 Mbp region, on a BAC clone OJ1174_H11, which also includes RM18639 - the most significant locus for the variation in FGP of the $\mathrm{F}_{2}$ population (Figure $3 \mathrm{~B}$ ).

\section{Discussion}

In the present study, we examined the major agronomic traits and grain/flour physicochemical properties of Suweon 542, a floury mutant rice line suitable for dry milling, and identified the chromosomal location responsible for the floury endosperm characteristics.

The particle size and damaged starch content of grain flours are determined during the milling process. They affect flour and starch characteristics such as gelatinization and pasting properties, solubility, swelling power, and digestibility, which have great influence on the quality of processed food products (Li et al. 2013). For rice flour, the quality of bread, noodles, and frying batters deteriorates when the raw flour has a coarse particle size and high damaged starch content (Araki et al. 2009; Lee and Lee 2006; Lee et al. 2013; Heo et al. 2012). Therefore, wet milling of polished rice has been widely used to reduce grain hardness through soaking procedures, which produces finer rice flour with less starch damage compared with dry milling. However, wet milling has a high production cost and raises environmental issues such as water waste and pollution caused by soaking procedures, hindering the expansion of the processed rice food industry in Korea. Hence, there is considerable demand for new rice cultivars suitable for dry milling.

Compared with the wild type and other rice cultivars, Suweon 542 demonstrated significantly lower grain hardness, smaller flour particle size, and lower damaged starch content when flour was produced from the dry milling of dehulled kernels (Table 2). It also showed a particle size distribution similar to that of a commercial wheat cultivar (Keumkang), which distinguished it from other rice cultivars (Table 3). These results were similar to those of Homma et al. (2007), who found that Hokuriku 166, a floury rice mutant, had finer flour than Koshihikari under both wet- and dry milling processes. Also, Ashida et al. (2010) reported that bread made from pin milled (dry milling) flour of milky-white mutants showed comparable quality to that made from jet milled (wet milling) flour of
Koshihikari. Likewise, Suweon 542 could be used as a valuable breeding source for improving the quality of rice flour produced by dry milling. Polished rice is generally used to produce flour in both dry and wet milling. In this study, we demonstrated the superior flour quality of Suweon 542 when the flour was produced by dry milling of dehulled kernels without polishing. Therefore, Suweon 542 could greatly reduce milling costs by eliminating the need to polish rice grains and using existing wheat mill facilities instead of establishing separate mills for rice flour production.

Notably, the agronomic traits of Suweon 542 were also favorable for its practical use in the processed rice food industry. The yield decrease of Suweon 542 due to the low grain weight of floury endosperm seeds was largely compensated for by an increase in the number of spikelets per panicle (Table 1). In addition, Suweon 542 maintained the early maturity of the wild type, Namil, which can be used in rice-wheat double cropping systems in Korea for improved arable land use. One possible drawback of Suweon 542 is the high rate of viviparous germination under prolonged rainfall during the harvesting season (data not shown). To overcome vivipary, late-planting cultivation could be practiced to avoid the rainy period during the ripening and harvesting season. Genetically, we are crossing Suweon 542 with indica germplasm that has high dormancy to alleviate preharvest sprouting. Also, additional screening of a mutant population of Suweon 542 is being carried out to identify floury mutant lines with high sensitivity to ABA (Schramm et al. 2013).

The observed FGP in Milyang23 (8.8\%) should be considered as a varietal characteristic, because the degree of chalkiness is a complex quantitative genetic trait and it varies greatly among cultivars and among environments within a cultivar (Patindol and Wang 2003; Liu et al. 2010). Indeed, association analysis detected a putative minor locus on chromosome 12, RM8215 $\left(R^{2}=0.150\right)$, in which the allele type of Milyang 23 was favorable to increase FGP. It was expected that co-segregation of the minor locus with $f l o 7(t)$ would affect the phenotype performance of the $F_{2}$ individuals to some extent. In such case, qualitative interpretations on the phenotypes, to infer genotypes of segregating progenies at the target locus, might cause serious bias per se, due to the phenotypic mis-scoring and environmental effect (Tabien et al. 2002). Therefore, we conducted association analysis between the FGP and marker genotypes of $F_{2}$ individuals by conducting single-locus ANOVA, since it is less sensitive to even modest numbers of phenotypic mis-scores (Jeung et al. 2007). Consequently, despite of the small $F_{2}$ population size $(N=94)$, association analysis with selective markers for the putative chromosomal regions and plotting of $F$ values from single-locus ANOVA provided 
not only estimated genetic effects but also the unbiased region of $f l o 7(t)$ on chromosome 5 (Table 4, Figure 3).

On chromosome 5, flo- 1 and flo4 were previously reported to control floury endosperm in rice. Although flo- 1 was localized to chromosome 5 by trisomic analysis, no information is available giving a more detailed location (Satoh and Omura 1981; Kaushik and Khush 1991). The floury endosperm mutant, flo4 was generated by knockout mutations in the $\mathrm{C}_{4}$-type pyruvate orthophosphate dikinase gene (OsPPDKB) (Kang et al. 2005), and locates within a BAC clone OJ1174_H11 (Figure 3B). By using the $94 \mathrm{~F}_{2}$ individuals, in the present study, the most highly likely region for $f l o 7(t)$ was determined on chromosome 5 as the $3.8 \mathrm{cM}$ interval (97.8-100.6 cM; Figure $3 \mathrm{~A}$ ), which is corresponding to the $530 \mathrm{Kbp}$ physical region (19.33-19.86 Mbp; Figure 3B). The $530 \mathrm{Kbp}$ physical region includes the BAC clone OJ1174_H11 harboring the most significant locus, RM18639, for the variation in FGP of the $\mathrm{F}_{2}$ population (Table 4; ANOVA). The result strongly suggests that $f l o 7(t)$ is physically linked to flo4, or might be another mutated allele of the OsPPDKB gene.

Kang et al. (2005) described the mutant phenotype of flo4 as 'white-core' endosperm with a normal outer portion. As the determined effects of the flo4 mutant on rice endosperm, they also characterized the aberrant physicochemical properties as 1) reduced kernel weight, 2) decreased amylose content, 3) increased protein content, and 4) significantly increased total lipid content. Unlike their description, Suweon 542 had an entirely white opaque kernel except for a thin peripheral area (Figure 1B). Moreover, Suweon 542 was significantly different from Namil (wild type) in terms of reduced protein content and increased amylose content, while the reduced kernel weight was consisted with flo4 (Table 2). Ashida et al. (2009) classified chalky rice mutant lines into 'white-core', having an opaque area only in the central region of the grain, and 'milky-white', with an entirely opaque grain except for the peripheral area, and observed that, when dry milled, the milky-white lines had significantly smaller flour particle size and lower damaged starch content compared with the white-core lines. While flo4 had white-core characteristics with a central chalky area (Kang et al. 2005), Suweon 542 showed milky-white characteristics with an overall opaque endosperm, so might be more suitable for dry milling (Figure 1, Table 3).

To efficiently transfer the floury endosperm characteristics of Suweon 542 to other commercial rice cultivars, it is essential to develop DNA marker tightly linked to the target gene, $f l o 7(t)$. Because Suweon 542 is a mutant line induced by sodium azide (Shin et al. 2009), it is desirable that the DNA marker depends on the authentic DNA polymorphism between Suweon 542 and Namil (wild type) followed by 1) fine physical mapping for FLO7 locus,
2) map-based cloning of the gene responsible for flo7 mutant, and 3) verifying the gene through complementing flo7 mutant line with transgenic line harboring a complete wild-type copy of FLO7. Currently, by using $\mathrm{F}_{2: 3}$ progeny lines derived from the heterozygous $F_{2}$ individuals at RM18639, construction of a segregating population is in progress to conduct fine mapping of flo7 locus, as well as to verify whether $f l o 7(t)$ is another mutated allele of $O s P P D K B$ as flo4 or a different gene with close proximity to OSPPDKB.

\section{Conclusions}

The floury endosperm of Suweon 542 makes it suitable for dry milling with a small flour particle size and low damaged starch content. Through genetic analysis on the $F_{2}$ progenies derived from Suweon 542/Milyang 23, a floury endosperm locus, $f l o 7(t)$, was located on chromosome 5 as a major recessive genetic factor directing floury endosperm of Suweon 542. Further physical mapping of $f l o 7(t)$ would facilitate efficient breeding of rice cultivars with proper dry milling adaptability that can be used in the processed rice food industry. Based on our studies, Suweon 542 was patented in Korea with its designated name, 'Namil(SA)-flo2' (Jeung et al. 2012).

\section{Methods}

\section{Plant materials and DNA extraction}

Suweon 542 is a floury endosperm mutant line derived from sodium azide treatment on a high-yield, early maturing, and non-glutinous japonica rice cultivar, Namil (Shin et al. 2009). In brief, after harvesting a single panicle from each $M_{1}$ plant, the ear-to-row method was used to advance generations with fertile individuals until the $\mathrm{M}_{7}$ generation. A total of 5,135 lines $\left(\mathrm{M}_{8}\right)$ with homogeneous phenotypes were eventually established as a mutant stock of Namil. To identify floury mutants with white opaque endosperm that crumble easily into a fine powder (Kaushik and Khush 1991), dehulled kernels (brown rice) were screened followed by I-KI staining to exclude waxy and dull mutants. Out of three floury mutants, the line with the pedigree 'Namil(SA)M2-1509-RGA-1-1-1-1', was designated as 'Namil(SA)-flo2'. After conducting local adaptation tests, the mutant line was also registered, as a Korean elite rice line, 'Suweon 542'.

To evaluate major agronomic traits and grain/flour physicochemical properties, Suweon 542 was cultivated with Namil (wild type, non-glutinous japonica cultivar), Hwaseong (non-glutinous japonica cultivar), and Seolgaeng (non-glutinous opaque endosperm japonica cultivar; Hong et al. 2011) in the experimental plot of the National Institute of Crop Science (NICS), Rural Development Administration (RDA), Suwon, Korea. During dry milling suitability tests, the harvesting of a Korean wheat cultivar, Keumkang (Park et al. 2011), was included as a control. 
A total of $94 \mathrm{~F}_{2}$ progenies from a cross between Suweon 542 and Milyang 23, a non-glutinous Tongil-type (derived from an indica-japonica cross) cultivar, were used for genetic studies on the induced floury endosperm characteristics. The parents and their $F_{2}$ progenies were grown by conventional methods in the experimental plot of the NICS, RDA. Total genomic DNA was extracted from fresh leaf tissue of the filed-grown $\mathrm{F}_{2}$ and parental lines according to Murray and Thompson (1980), with minor modifications.

\section{Evaluation of agronomic traits and grain/flour physicochemical properties}

Replicated yield trials (RYT) were conducted to evaluate major agronomic traits as well as grain/flour physicochemical properties in the field at NICS, RDA, Suwon, Korea in 2010. The seeds of each rice line were sown on April 25, and were transplanted on May 25 under a randomized complete block design (RCBD) with three replication plots. Each plot, consisting of eight rows with 30 hills per row and three plants per hill, was planted with $30 \times 15 \mathrm{~cm}$ spacing. Rice lines were nursed and evaluated for major agronomic traits following the standard evaluation method for rice (RDA, Korea 2003). The amount of fertilizer application was $90-45-57 \mathrm{Kg} /$ ha for $\mathrm{N}-\mathrm{P}_{2} \mathrm{O}_{5}-\mathrm{K}_{2} \mathrm{O}$, and the 10 hills in the middle rows were used to determine days-to-heading (HD), culm length (CL), panicle length (PL), tiller number (TN), spikelet number per panicle $(\mathrm{SN})$, and ripened grains percentage (RGP). Grain yield per plot was evaluated based on a grain harvest of 100 hills in the central row of each plot. Brown rice (dehulled kernels) yield (BRY; ton/ha) was then calculated based on the brown rice recovery rate. The 1,000grain weight (TGW) was measured in grams as the average weight of 1,000 fully filled brown rice grains from each plot.

The grain hardness of brown rice was determined by measuring the pressure at grain breakage point using a 5-mm probe attachment of TA.XT Plus (Stable Micro Systems, Godalming, Surrey, UK) under the conditions of $0.4 \mathrm{~mm} / \mathrm{sec}$ and $40.0 \mathrm{~g}$ for test speed and trigger force, respectively. The cleaved endosperm surface was observed with an S-550 field emission scanning electron microscope (Hitachi Hi-Tech, Tokyo, Japan).

After tempering, the dehulled kernels of Suweon 542 and other rice cultivars were milled using a Buhler MLU202 laboratory mill (Buhler AG, Uzwil, Switzerland) with Keumkang, a Korean wheat cultivar, as a control. An extraction of about $60 \%$ was prepared by blending the first and second break millstreams and the first and second reduction millstreams to examine physicochemical properties. Particle size distribution in the flour was determined using a LS13320 laser diffraction particle size analyzer (Beckman Coulter, CA, USA). Five grams of flour was transferred into the laser diffraction particle size analyzer's dispersion tube for size measurement. Damaged starch content was evaluated with a starch damage assay kit (Megazyme International Ireland, Wicklow, Ireland) following the manufacturer's instructions. The lightness of rice flour was measured with a JS-555 (Color Techno System, Tokyo, Japan). Moisture, protein, and ash contents of the rice flour were determined following the 44-15A, 46-30, and 08-01 methods of AACC (AACC American Association of Cereal Chemists 2000). The amylose content of the rice flour was estimated by the method of Juliano (1971).

\section{Genetic studies}

Dehulled kernels of parental lines and $F_{2: 3}$ seeds harvested from the $94 \mathrm{~F}_{2}$ individuals were investigated in terms of floury grains percentage (FGP), as the phenotypic data for genetic analysis. Randomly selected 150-200 seeds were dehulled to evaluate FGP by visual inspection, where a dehulled kernel with more than $50 \%$ opaque area was considered as 'floury'

A total of 70 SSR markers displaying polymorphism between Suweon 542 and Milyang 23 were selected as anchor markers after careful comparisons with previous reports in terms of the band size of amplified products (McCouch et al. 2002). The $\mathrm{F}_{2}$ mapping population of 94 individuals was genotyped at the loci tagged by the preselected anchor markers to construct a linkage map skeleton. To determine the relative physical positions of the SSR markers, their primer sequences were projected over a reference rice genome (e-Landing; Jeung et al. 2007), Os-Nipponbare-Reference-IRGSP-1.0 (http://rapdb. dna.affrc.go.jp\%/download/irgsp1.html), using the 'BLAST' menu at the Rice Annotation Project database (RAP-DB) (http://rapdb.dna.affrc.go.jp/tools/blast). When both primer sequences successfully recognized their physical locations for annealing, the expected PCR product size was calculated to judge the PCR products. Based on the defined physical locations, the corresponding $\mathrm{BAC} / \mathrm{PAC}$ clones were identified and their determined cM positions were directly adopted from the IRGSP Build5 Pseudomolecules (http://rgp.dna.affrc.go.jp/\%E/IRGSP/Build5/ \%build5.html). After identifying rice chromosome 5 as the location of the most significant genetic factor for floury endosperm characteristics, 14 additional SSR markers were further incorporated into the linkage map skeleton to increase marker density (see Table 4; Locus, Ap = 2, 3).

PCR was performed in a total volume of $30 \mu$ l containing $10 \mathrm{ng}$ of DNA template, $10 \mathrm{pmol}$ of each primer, $1.5 \mathrm{mM}$ of $\mathrm{MgCl}_{2}, 0.2 \mathrm{mM}$ of dNTPs, and $1 \mathrm{U}$ of Taq polymerase (Nurotics, Daejeon, Korea). PCR began with one cycle at $95^{\circ} \mathrm{C}$ for $3 \mathrm{~min}$, followed by 40 cycles of $95^{\circ} \mathrm{C}$ for $30 \mathrm{~s}, 55^{\circ} \mathrm{C}$ for $30 \mathrm{~s}$, and $72^{\circ} \mathrm{C}$ for $1 \mathrm{~min}$, with a final extension at $72^{\circ} \mathrm{C}$ for $10 \mathrm{~min}$ (MJ Research PTC-100 
thermocycler; Waltham, MA, USA). The primer annealing temperature for SSR markers was $55^{\circ} \mathrm{C}$. Sequencing gel electrophoresis $(5 \%$ polyacrylamide, $6 \mathrm{M}$ urea, $1 \times \mathrm{TBE}$, $80 \mathrm{~W})$ was used for SSR-PCR product separation and bands were visualized using Silver Sequence ${ }^{\mathrm{TM}}$ (Promega, Madison, WI, USA).

Chi-square analysis was conducted for segregation tests on the observed number of floury kernels and the revealed genotypes of $F_{2}$ individuals for each SSR locus. PROC UNIVARIATE and PROC GLM of the SAS statistical package (Version 8.1, SAS institute, Cary, NC, USA) were used to calculate descriptive statistics on FGP of the $F_{2: 3}$ seeds, and to estimate the relative contribution of tested loci to floury grains percentage (single-locus ANOVA), respectively. For the $F$-test of loci, markers having a $P$ value less than 0.05 were declared to be significant empirically. The percentage of phenotypic variation explained $\left(R^{2}\right)$, additive genetic effect, and the degree of dominance were then estimated for the declared loci within the mapping population. Judging the predetermined SSR marker orders, through $e$-Landing, on the linkage map of chromosome 5, and estimating the practical recombination fractions between SSR marker pairs were conducted with the 'ripple' and 'map' commands of the computer software MAPMAKER/EXP3.0 (Lincoln et al.1992). The marker intervals were calculated by using the Kosambi mapping function.

\section{Competing interests}

The authors declare that they have no competing interests.

\section{Authors' contributions}

YJM and JUJ conducted the genetic analysis to localize the genetic locus directing the floury character. JUJ and YSS developed the mutant population of Namil and selected Suweon 542, followed by evaluations of agronomic traits. CSP, KHK, and BKK carried out the experiments to address the physicochemical characteristics of Suweon 542. All authors read and approved the final manuscript.

\section{Acknowledgements}

This research was supported by grants from Next-Generation BioGreen21 (PJ008160).

\section{Author details}

${ }^{1}$ National Institute of Crop Science, Rural Development Administration, Suwon 441-857, Republic of Korea. '2Department of Crop Science and Biotechnology, Chonbuk National University, Jeonju 561-756, Republic of Korea.

Received: 25 July 2013 Accepted: 5 December 2013 Published: 9 December 2013

\section{References}

AACC (American Association of Cereal Chemists) (2000) Approved methods of the AACC, 10th edn. The Association, St. Paul, Minnesota

Altheide MC, Morawicki RO, Hager TJ (2012) Impact of milling and water-to-rice ratio on cooked rice and wastewater properties. Food Sci Technol Int 18(3):291-298

Araki E, Ikeda TM, Ashida K, Takata K, Yanaka M, lida S (2009) Effects of rice flour properties on specific loaf volume of one-loaf bread made from rice flour with wheat vital gluten. Food Sci Technol Res 15(4):439-448

Ashida K, lida S, Yasui T (2009) Morphological, physical, and chemical properties of grain and flour from chalky rice mutants. Cereal Chem 86(2):225-231
Ashida K, Araki E, lida S, Yasui T (2010) Flour properties of milky-white rice mutants in relation to specific loaf volume of rice bread. Food Sci Technol Res 16(4):305-312

Demirkesen I, Sumnu G, Sahin S (2013) Image analysis of gluten-free breads prepared with chestnut and rice flour and baked in different ovens. Food Biopreocess Technol 6:1749-1758

Hasjim J, Li E, Dhital S (2013) Milling of rice grains: Effects of starch/flour structures on gelatinization and pasting properties. Carbohydrate Polymers 92(1):682-690

Heo S, Lee SM, Shim JH, Yoo SH, Lee S (2012) Effect of dry- and wet milled rice flours on the quality attributes of gluten-free dough and noodles. J Food Eng 116(1):213-217

Homma N, Morohashi K, Yoshii Y, Hosokawa H, Miura K (2007) Properties and components of the floury and sugary mutant rice cultivars developed in the Hokuriku region. Food Sci Technol Res 13(4):422-426

Hong HC, Moon HP, Choi HC, Hwang HG, Kim YG, Kim HY, Yea JD, Shin YS, Kang KH, Choi YH, Cho YC, Baek MK, Yang Cl, Choi IS, Ahn SN, Yang SJ (2011) A lodging tolerant, opaque rice cultivar 'Seolgaeng'. Kor J Breed Sci 43(6):532-537

Hong HC, Choi HC, Hwang HG, Kim YG, Moon HP, Kim HY, Yea JD, Shin YS, Choi YH, Cho YC, Baek MK, Lee JH, Yang Cl, Jeong KH, Ahn SN, Yang SJ (2012a) A lodging-tolerance and dull rice cultivar 'Baegjinju'. Kor J Breed Sci 44(1):51-56

Hong HC, Kim YG, Choi YH, Yang SJ, Lee KS, Lee JH, Jung OY, Yang Cl, Cho YC, Choi IS, Baek MK, Kim MK, Yea JD, Hwang HG, Roh JH, Kim SL, Choi HC, Lee YT, Lee SH (2012b) A medium-maturing, giant-embryo, and germination brown rice cultivar 'Keunnun'. Kor J Breed Sci 44(2):160-164

Jeung JU, Kim BR, Cho YC, Han SS, Moon HP, Lee YT, Jena KK (2007) A novel gene, Pi4O(t), linked to the DNA markers derived from NBS-LRR motifs confers broad spectrum of blast resistance in rice. Theor Appl Genet 115(8):1163-1177

Jeung JU, Shin YS, Park CS, Choi IS, Kang KH (2012) A floury japonica rice line, Namil (SA)-flo2, suitable for dry milling process and the food compositions containing Namil(SA)-flo2 as an active compositions. Korea Patent 10-1190642:8

Juliano BO (1971) A simplified assay for milled-rice amylose. Cereal Sci Today 16(10):334-340

Jun HI, Yang EJ, Kim YS, Song GS (2008) Effect of dry and wet millings on physicochemical properties of black rice flours. J Korean Soc Food Sci Nutr 37(7):900-907

Kadan RS, Bryant RJ, Boykin DL (2001) Effect of processing conditions on qualities of rice fries. J Food Sci 66(4):610-613

Kang HG, Park S, Matsuoka M, An G (2005) White-core endosperm floury endosperm-4 in rice is generated by knockout mutations in the $\mathrm{C}_{4}$-type pyruvate orthophosphate dikinase gene (OsPPDKB). Plant J 42(6):901-911

Kang SY, Shin IC, Kim DS, Lee GJ, Kim JB, Lee DY, Lee SY, Lee DJ (2008) A new green-kerneled glutinous rice mutant variety, "Nogwonchalbyeo" developed by gamma ray irradiation. Kor J Breed Sci 40(3):303-307

Kaushik RP, Khush GS (1991) Genetic analysis of endosperm mutants in rice Oryza sativa L. Theor Appl Genet 83(2):146-152

Kim MR (2011) The status of Korea's rice industry and the rice processing industry. Food Industry and Nutriton 16(1):22-26

Kim RY, Kim CS, Kim HI (2009) Physicochemical properties of non-waxy rice flour affected by grinding methods and steeping times. J Korean Soc Food Sci Nutr 38(8):1076-1083

Lee YT, Kim Y (2011) Physicochemical properties of brown rice flours differing in amylose content prepared by different milling methods. J Korean Soc Food Sci Nutr 40(12):1797-1801

Lee $\mathrm{MH}$, Lee YT (2006) Bread-making properties of rice flours produced by dry, wet and semi-wet milling. J Korean Soc Food Sci Nutr 35(7):886-890

Lee SM, Yoo J, Inglett GE, Lee S (2013) Particle size fractionation of high-amylose rice (Goami 2) flour as an oil barrier in a batter-coated fried system. Food Bioprocess Technol 6(3):726-733

Li E, Dhital S, Hasjim J (2013) Effects of grain milling on starch structures and flour/starch properties. Starch/Stärke. doi:10.1002/star.201200224

Lincoln S, Daly M, Lander ES (1992) Constructing Genetic Maps with MAPMAKER/ EXP 3.0. Whitehead Institute Technical Report, Cambridge, Massachusetts

Liu X, Guo T, Wan X, Wang H, Zhu M, Li A, Su N, Shen Y, Mao B, Zhai L, Wan J (2010) Transcriptome analysis of grain-filling caryopses reveals involvement of multiple regulatory pathways in chalky grain formation in rice. BMG Genomics 11:730

McCouch SR, Teytelman L, Xu Y, Lobos KB, Clare K, Walton M, Fu B, Maghirang R, Li Z, Xing Y, Zhang Q, Kono I, Yano M, Fjellstrom R, DeClerck G, Schneider D, Cartinhour S, Ware D, Stein L (2002) Development and mapping of 2240 new SSR markers for rice (Oryza sativa L.). DNA Res 9(6):199-207 
Murray MG, Thompson WF (1980) Rapid isolation of high molecular weight plant DNA. Nucleic Acids Res 8:4321-4325

Nishio T, lida S (1993) Mutants having a low content of 16-kDa allergenic protein in rice (Oryza sativa L.). Theor Appl Genet 86(2-3):317-321

Park CS, Kang CS, Jeung JU, Woo SH (2011) Influence of allelic variations in glutenin on the quality of pan bread and white salted noodles made from Korean wheat cultivars. Euphytica 180(2):235-250

Patindol J, Wang Y (2003) Fine structure and physicochemical properties of starches from chalky and translucent rice kernels. J Agric Food Chem 51:2777-2784

Qiao Y, Lee SI, Piao R, Jiang W, Ham TH, Chin JH, Piao Z, Han L, Kang SY, Koh HJ (2010) Fine mapping and candidate gene analysis of the floury endosperm gene, FLO (a), in rice. Mol Cells 29(2):167-174

RDA (Rural Development Administration) (2003) Manual for standard evaluation method in agricultural experiment and research. RDA, Suwon (Korea), p 838

Ryoo N, Yu C, Park CS, Baik MY, Park IM, Cho MH, Bhoo SH, An G, Hahn TR, Jeon JS (2007) Knockout of a starch synthase gene OsSSIIla/Flo5 causes white-core floury endosperm in rice (Oryza sativa L.). Plant Cell Rep 26(7):1083-1095

Satoh H, Omura T (1981) New endosperm mutations induced by chemical mutagens in rice, Oryza sativa L. Japan J Breed 31(3):316-326

Schramm EC, Nelson SK, Kidwell KK, Steber CM (2013) Increased ABA sensitivity results in higher seed dormancy in soft white spring wheat cultivar 'Zak'. Theor Appl Genet 126(3):791-803

She KC, Kusano H, Koizumi K, Yamakawa H, Hakata M, Imamura T, Fukuda M, Naito N, Tsurumaki Y, Yaeshima M, Tsuge T, Matsumoto K, Kudoh M, Itoh E, Kikuchi S, Kishimoto N, Yazaki J, Ando T, Yano M, Aoyama T, Sasaki T, Satoh $\mathrm{H}$, Shimada H (2010) A novel factor FLOURY ENDOSPERM2 is involved in regulation of rice grain size and starch quality. Plant Cell 22(10):3280-3294

Shin YS, Park CS, Seo YW, Jeung JU (2009) Characteristics of endosperm starch of the rice mutant lines induced by sodium azide. Kor J Breed Sci 41(2):84-91

Singh N, Kaur L, Sodhi NS, Sekhon KS (2005) Physicochemical, cooking and textural properties of milled rice from different Indian rice cultivars. Food Chem 89:253-259

Statistics Korea (2013) http://kostat.go.kr/portal/korea/index.action. Accessed 4 Jun 2013

Tabien RE, Li Z, Paterson AH, Marchetti MA, Stansel JW, Pinson SRM (2002) Mapping QTLs for Weld resistance to the rice blast pathogen and evaluating their individual and combined utility in improved varieties. Theor Appl Genet 105:313-324

Tran TTB, Shelat KJ, Tang D, Li E, Gilbert RG, Hasjim J (2011) Milling of rice grains. The degradation on three structural levels of starch in rice flour can be independently controlled during grinding. J Agric Food Chem 59(8):3964-3973

Zhang D, Wu J, Zhang Y, Shi C (2012) Phenotypic and candidate gene analysis of a new floury endosperm mutant (osagp/2-3) in rice. Plant Mol Biol Rep 30(6):1303-1312

Zhou Z, Robards K, Helliwell S, Blanchard C (2002) Composition and functional properties of rice. Int J Food Sci Technol 37:849-868

doi:10.1186/1939-8433-6-37

Cite this article as: Mo et al:: Agronomic and genetic analysis of Suweon 542, a rice floury mutant line suitable for dry milling. Rice 2013 6:37.

\section{Submit your manuscript to a SpringerOpen ${ }^{\circ}$ journal and benefit from:}

- Convenient online submission

- Rigorous peer review

- Immediate publication on acceptance

- Open access: articles freely available online

- High visibility within the field

- Retaining the copyright to your article

Submit your next manuscript at $\gg$ springeropen.com 